\title{
Perspectives and Potential Applications of Ruthenium-Based Nanocarriers for Cancer Therapy
}

\author{
Rita Santamaria ${ }^{* 1}$, Carlo Irace ${ }^{1}$, Gerardino D’Errico ${ }^{2}$, Daniela Montesarchio ${ }^{2}$ and Luigi Paduano ${ }^{2}$ \\ ${ }^{1}$ Department of Pharmacy, University of Napoli "Federico II", via D. Montesano 49, I-80131, Napoli, Italy \\ ${ }^{2}$ Department of Chemical Sciences, University of Napoli "Federico II", Complesso Universitario di Monte \\ Sant'Angelo, via Cintia 21, I-80126, Napoli, Italy
}

${ }^{*}$ Corresponding author: Rita Santamaria, Associate Professor, Department of Pharmacy, University of Napoli “Federico II”, via D. Montesano 49, I-80131, Napoli, Italy, Fax: 0039081678403, Tel: 0039081678421, E-Mail: rsantama@unina.it

Citation: Rita Santamaria, Carlo Irace, Gerardino D’Errico, Daniela Montesarchio, Luigi Paduano (2013) Perspectives and Potential Applications of Ruthenium-Based Nanocarriers for Cancer Therapy. J Pharm Drug Devel 1(2): e201. doi: 10.15744/2348-9782.1.e201

\section{Received Date: September 30, 2013 Accepted Date: October 15, 2013 Published Date: October 23, 2013}

Cancer is a highly heterogeneous disease characterized by continuous uncontrolled growth and expansion of abnormal cells [1]. In general, in tumor cells the signaling pathways regulating cellular processes, as cell growth and division and cell-to-cell communication result strongly altered. Furthermore, the cancer cells accumulate repeated mutations that provide a selective growth advantage over other cells. In addition, some cancer cells become invasive and then metastasize. This characteristic together with the genetic and phenotypic heterogeneity of the tumor cells makes cancer disease particularly difficult to treat and eradicate.

In order to obtain more efficacious anticancer treatments, novel therapeutic approaches are being explored to replace conventional therapies, such as surgery, radiotherapy, and chemotherapy. In this framework the cancer nanotechnologies, although still in development phase, have demonstrated a great therapeutic potential. In fact, the nanotechnology-based drug-delivery systems have several advantages over conventional therapies, such as longer half life, enhancement in biodistribution and reduced toxic side effects. So far, several nanotechnological-based systems have been developed for cancer treatment, including inorganic, polymeric, and hybrid carriers [2,3]. Currently used inorganic nanocarriers are quantum dots, carbon nanotubes, and gold nanoparticles. Quantum dots are colloidal nanoparticles that display fluorescence spectra and their surface can be modified to improve sensitivity, specificity, and visualization in target tissue [4]. They are promising candidates for oncological imaging applications, though further studies on their toxicity are required [5]. Carbon nanotubes are particularly attractive as nanocarriers due to their high stability, easy bioconjugation and simplicity of manufacture [6]; however, their utilization in oncology is limited because of their toxicity that still remains a controversial question. Gold nanoparticles have been used in the last decade and have obtained special attention for their biocompatibility and easy conjugation to biomolecules, properties that make them largely suitable for applications in anticancer therapy $[7,8]$. In recent years, gold nanoparticles have been also explored with success in theranostic applications, an integration of therapeutics with diagnostics particularly useful for monitoring patients' response to therapy [9].

Among nanoparticles, the polymeric nanocarriers have specific features, such as biocompatibility, biodegradability, and high drug loading capacity, that render them appropriate as antineoplastic agents. In fact, polymeric particles allow to encapsulate a large variety of drugs and release them over prolonged periods; furthermore, they can be modified with multiple ligands and are particularly stable. There are various forms of polymeric nanocarriers, such as dendrimers, micelles and liposomes. Dendrimers are characterized by a central core, an internal region and numerous terminal groups. Chemical modifications of their terminal groups render dendrimers excellent drugs [10]. Polymeric micelles are amphiphilic spherical structures composed of a hydrophobic core that stores up the drug and a hydrophilic shell that stabilizes the micelle in aqueous environment. The micelles are highly stable, very biocompatible and suitable for drug delivery and controlled drug release [11]. Liposomes are globular nanostructures composed of one or more amphiphilic lipid bilayers [12]. They have high biocompatibility because of their composition similar to the cell membranes. A particular feature of liposomes is that they possess distinct hydrophilic and hydrophobic regions, allowing to encapsulate both waterinsoluble and water-soluble drugs. Moreover, the liposome surface can be modified with ligands or polymers to enhance drug delivery specificity.

One of the limitations emerged during the therapeutic use of nanoparticles is the short plasma half-life due to the opsonization process that yields their degradation by reticuloendothelial system. To overcome this problem nanocarriers have been modified to prolong their circulation and increase drug efficacy. In particular, the conjugation with hydrophilic polymers, such as Polyethylene Glycol (PEG), generates a stealth surface from opsonization and decreases their susceptibility to metabolic enzymes [13]. 
In recent decades the scientific community has proposed an increasing number of antineoplastic agents. Among these, transition metal-based complexes represent a very important class of chemotherapeutics, intensively used for clinical treatments; the field of metal-based anticancer drugs has been until now dominated by the precious metal platinum. The antineoplastic activity of Cisplatin was discovered in 1969 [14] and approved for cancer therapy in 1978, soon becoming a reference drug in the treatment of many solid tumors $[15,16]$. Some analogues of Cisplatin have been tested and then approved as drugs, such as Carboplatin and Oxaliplatin $[17,18]$. Whilst the chemotherapeutic success of platinum is indubitable, it is not the perfect drug. Indeed, it is not effective against some common types of cancer and many metastatic lesions, drug resistance is common and it has a range of side effects. These and other limitations have prompted researchers to develop more effective and less toxic metal-based anticancer agents.

In recent years ruthenium complexes have attracted much interest as a promising alternative to platinum, showing a remarkable antitumoural and antimetastatic activity, associated with lower toxicity. Interestingly, ruthenium's properties are well suited towards pharmacological applications: it can access a range of oxidation states (II, III and IV) under physiologically relevant conditions, with relatively low energy barriers to interconversion between oxidation states, thus allowing for ready oxidation state changes when inside the cell. Furthermore, ruthenium can form strong chemical bonds with a range of different elements of varying chemical 'hardness' and electronegativities, allowing the metal to bind to a wide range of biomolecules.

Among ruthenium-based complexes, the first promising compounds have been studied in the early 90's, developing the ruthenium complex named NAMI-A (Figure 1), endowed with relevant anticancer activity $[19,20]$ This compound, along with KP1019, is presently in phase II of clinical trials [21,22]. However, one of the major drawbacks of ruthenium complexes is their rather limited stability in aqueous solutions. Despite the good outcome throughout advanced clinical evaluation showed by NAMI-A and KP1019, this is a central issue that has required a reconsideration of the ruthenium complexes therapeutic effectiveness. Under physiological conditions, the degradation process is imputable to the replacement of chloride ions, as well as of the DMSO ligand, with water molecules and/or hydroxide ions, followed by the formation of poly-oxo species [23]. Although it is not yet clear whether this can really alter the ruthenium complexes anticancer activity, the premature aquation and hydrolysis of other anticancer drugs, as in the case of Cisplatin, can deactivate or activate the most of the administered complex [24]. For this reason, the design of long-life ruthenium-based antineoplastic agents is still a primary goal and consequently a large number of alternative ruthenium-based complexes have been designed in the recent years [25]. In this contest, basing on the design of novel amphiphilic nanovectors for drug delivery and aiming at improve the stability of the ruthenium-based drugs, as well as their cellular uptake and effectiveness, a new concept for ruthenium-based anticancer therapy has been proposed.
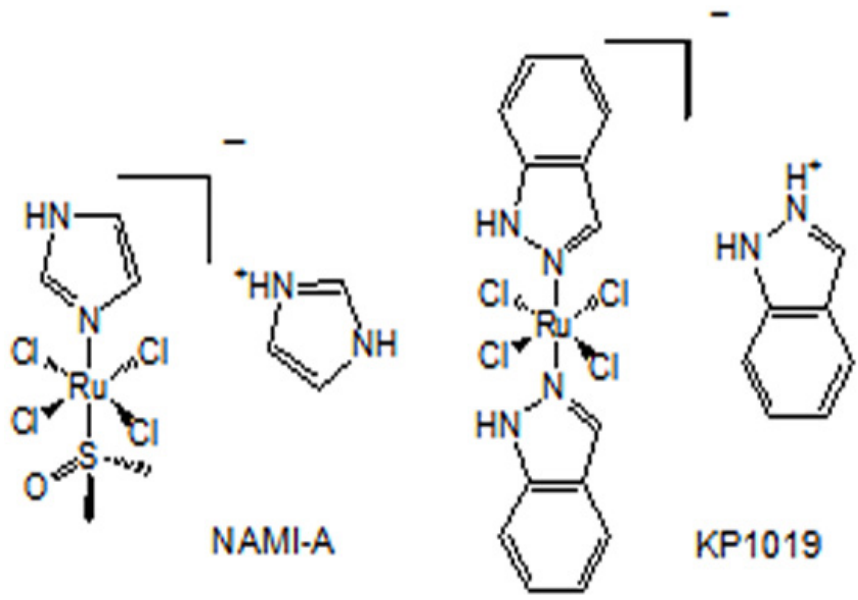

Figure 1: Chemical structures of NAMI-A and KP1019.

The basic idea consisted in incorporating the cytotoxic ruthenium complex into a highly functionalized molecular structure, ensuring both an efficient protection against degradation and a high cellular internalization of the metal. More in detail, a new NAMI-A analog, named AziRu (Figure 2), has been designed and synthesized showing higher in vitro cytotoxicity than NAMI-A itself; AziRu has been then inserted into an amphiphilic nucleolipidic scaffold, as the core structural motif attached to the nucleobase of uridine or thymidine, which were further decorated with one or two lipid residues and with one oligoethylene glycol chain of variable length [26]. Besides being capable to mimic the molecular organizations of the biological systems, nucleolipids have been selected as scaffold for the amphiphilic ruthenium complexes for the possibility to form a variety of supramolecular systems such as liposomes/vesicles, cubic phases, ribbons, etc. that have found an increasing application in the biomedical field [27]. In this way a series of novel amphiphilic ruthenium complexes able to form supramolecular aggregates have been synthesized and characterized; the structures of these complexes, named ToThyRu, HoThyRu and DoHuRu, are shown in Figure 2 and are based on a pyrimidine deoxyribo- (Thymidine, as in the case of ToThyRu and HoThyRu) or ribonucleoside (Uridine, for DoHuRu). A pyridine residue was attached to the nucleobase as a chelating moiety able to complex $\mathrm{Ru}$ (III) ions. The amphiphilic ruthenium complexes have been studied as pure aggregates, as well as in co-aggregation with the zwitterionic lipid 1-palmitoyl-2-oleoyl-sn-glycero-3phosphocholine (POPC) or the cationic lipid 1,2-dioleoyl3-trimethylammoniumpropane chloride (DOTAP). Indeed, the combination of $\mathrm{Ru}$ nucleolipidic complexes with phospholipids can allow a fine tuning of the metal amount to be administered, as well as protection from degradation, since the ruthenium complex is lodged in the liposome bilayer. Among phospholipids, POPC is of particular interest as a component of natural membranes, ensuring high biocompatibility to liposomes [28]. On the other hand, the co-aggregation of the amphiphilic ruthenium complexes with the cationic lipid DOTAP is aimed at enhancing their antineoplastic activity by increasing the ruthenium content within the nanoaggregates 


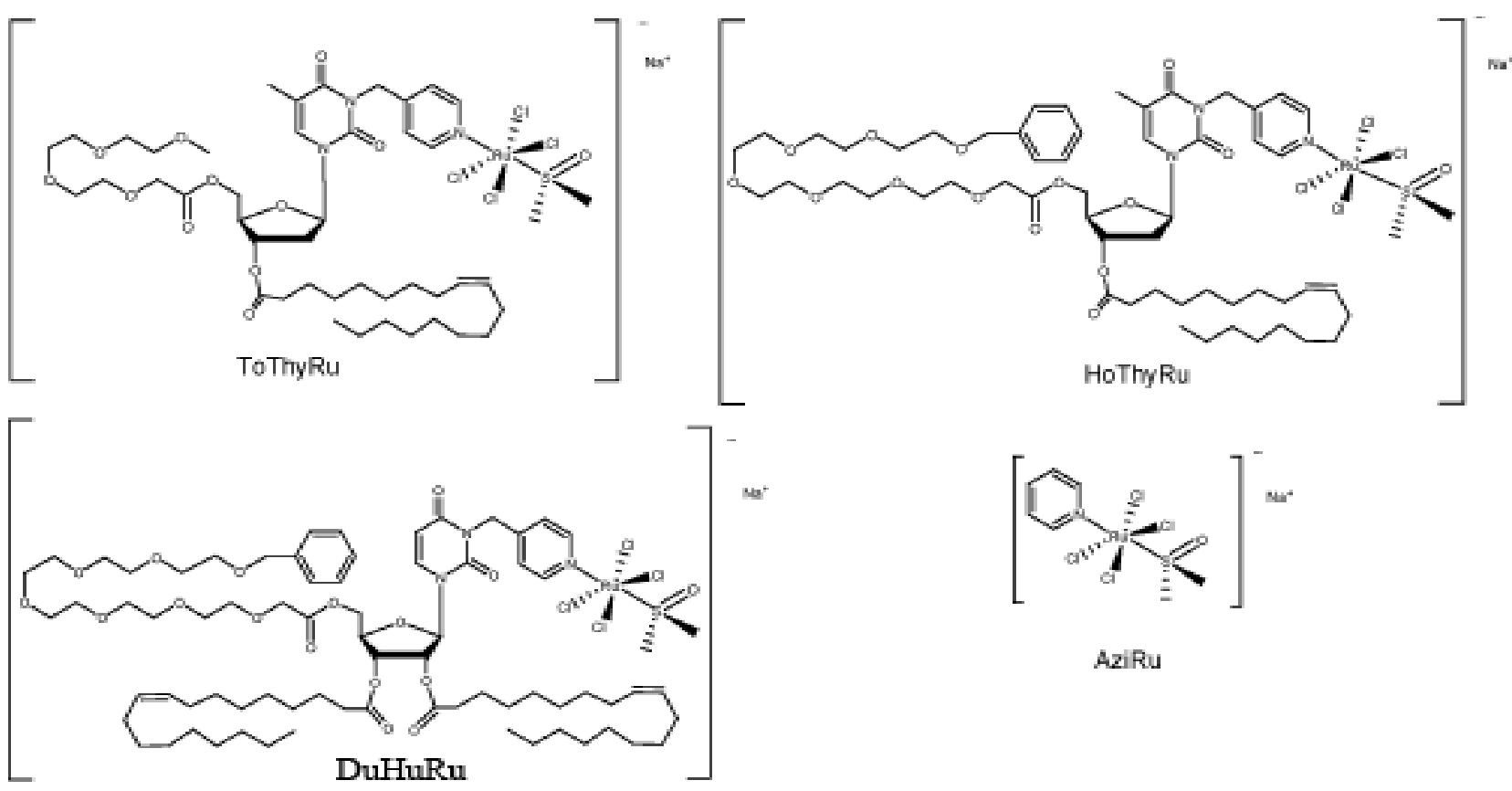

Figure 2: Molecular structures of the Ru(III) complexes ToThyRu, HoThyRu, DoHuRu and AziRu [26, 28].

and favoring their cell uptake [29]. Effective retardation of the ligand exchange processes in physiological solutions, resulting into formulations stable for months, has been obtained with the $\mathrm{Ru}(\mathrm{III})$ complex present up to a maximum ratio of $15 \%$ in mol in mixture with POPC, and up to $50 \%$ in mixture with

The Ru-containing nanoaggregates bioactivity has been explored on a panel of human and non-human cancer cell lines by fluorescence microscopy, to establish cellular uptake, and by in vitro bioscreenings to determine antitumor activity. All the results converge in showing high anticancer activities for all the ruthenium complexes formulations. In fact, the nucleolipid-based ruthenium complexes stabilized in POPC or in DOTAP lipid bilayer are more effective than AziRu, which in turn was found to have $\mathrm{IC}_{50}$ values lower than other known $\mathrm{Ru}$ (III) complexes, thereby exhibiting high in vitro antiproliferative activity as well as biocompatible properties and long life stability [29].

To the best of our knowledge, as part of the search for novel anticancer agents, these molecules are among the most promising ruthenium-based drugs currently described in the literature.. Taken together, these findings fully validate the highly functionalized nucleolipid-based design as an efficient and innovative strategy to produce a variety of suitable in vivo carriers for cytotoxic ruthenium complexes, and open the way to new concepts for metal-based drug delivery in cancer

\section{References}

1. Blanpain C (2013) Tracing the cellular origin of cancer. Nat Cell Biol 15:126134.

2. Zamboni WC, Torchilin V, Patri AK, Hrkach J, Stern S, et al. (2012) Best practices in cancer nanotechnology: perspective from NCI nanotechnology alliance. Clin Cancer Res 18:3229-3241.

3. Rahman M, Ahmad MZ, Kazmi I, Akhter S, Afzal M, et al. (2012) Advancement in multifunctional nanoparticles for the effective treatment of cancer. Expert Opin Drug Deliv 9:367-381.
4. Cai W, Chen X (2008) Preparation of peptide-conjugated quantum dots for tumor vasculature-targeted imaging. Nat Protoc 3:89-96.

5. Hauck TS, Anderson RE, Fischer HC, Newbigging S, Chan WC (2010) In vivo quantum-dot toxicity assessment. Small 6:138-144.

6. Hong H, Gao T, Cai WB (2009) Molecular imaging with single-walled carbon nanotubes. Nano Today 4:252-261.

7. Patra CR, Bhattacharya R, Mukherjee P (2010) Fabrication and functional characterization of gold nanoconjugates for potential application in ovarian cancer. J Mater Chem 20:547-554.

8. Patra CR, Bhattacharya R, Mukhopadhyay D, Mukherjee P (2010) Fabrication of gold nanoparticles for targeted therapy in pancreatic cancer. Adv Drug Deliv Rev 62:346-361.

9. Ahmad MZ, Akhter S, Rahman Z, Akhter S, Anwar M, et al. (2013) Nanometric gold in cancer nanotechnology: current status and future prospect. J Pharm Pharmacol 65:634-651.

10. Tekade RK, Kumar PV, Jain NK (2009) Dendrimers in oncology: an expanding horizon. Chem Rev 109: 49-87.

11. Osada K, Christie RJ, Kataoka K (2009) Polymeric micelles from poly(ethylene glycol)- poly(amino acid) block copolymer for drug and gene delivery. J R Soc Interface 6:S325-S339.

12. Jesorka A, Orwar O (2008) Liposomes: Technologies and analytical applications. Annu Rev Anal Chem 1:801-832.

13. Byrne JD, Betancourt T, Brannon-Peppas L (2008) Active targeting schemes for nanoparticle systems in cancer therapeutics. Adv Drug Deliv Rev 60:1615-1626.

14. Rosenberg B, VanCamp L, Trosko JE, Mansour VH (1969) Platinum compounds: a new class of potent antitumor agents. Nature 222:385-386.

15. Boulikas T, Vougiouka M (2003) Cisplatin and platinum drugs at the molecular level. Oncol Rep 10:1663-1682.

16. Rafique S, Idrees M, Nasim A, Akbar H, Athar A (2010) Transition metal complexes as potential therapeutic agents. Biotechnol Mol Biol Rev 5:38-45.

17. Graham J, Muhsin M, Kirkpatrick P (2004) Oxaliplatin. Nat Rev Drug Discov 3:11-12.

18. Kelland L (2007) The resurgence of platinum-based cancer chemotherapy. Nat Rev Cancer 7:573-584.

19. Bergamo A, Cocchietto M, Capozzi I, Mestroni G, Alessio E, et al. (1996) Treatment of residual metastases with $\mathrm{Na}$ [trans-RuCl4(DMSO)Im] and ruthenium uptake by tumor cells. Anti-Cancer Drugs 7:697-702.

20. Sava G, Gagliardi R, Bergamo A, Alessio E, Mestroni G (1999) Treatment of metastases of solid mouse tumours by NAMI-A: comparison with cisplatin, cyclophosphamide and dacarbazine. Anticancer Res 19:969-972. 
21. Lentz F, Drescher A, Lindauer A, Henke M, Hilger RA, et al. (2009) Pharmacokinetics of a novel anticancer ruthenium complex (KP1019, FFC14A) in a phase I dose-escalation study. Anti-Cancer Drugs 20:97-103.

22. Rademaker-Lakhai JM, van den Bongard D, Pluim D, Beijnen J H, Schellens JH (2004) A Phase I and pharmacological study with imidazoliumtransDMSO-imidazole-tetrachlororuthenate, a novel ruthenium anticancer agent. Clin Cancer Res 10:3717-3727.

23. Bouma M, Nuijen B, Jansen MT, Sava G, Flaibani A, et al. (2002) A kinetic study of the chemical stability of the antimetastatic ruthenium complex NAMIA. Int J Pharm 248:239-246.

24. Galanski M, Keppler BK (2007) Searching for the magic bullet: anticancer platinum drugs which can be accumulated or activated in the tumor tissue. Anti-Cancer Agents Med Chem 7:55-73.

25. Levina A, Mitra A, Lay PA. Recent developments in ruthenium anticancer drugs (2009) Metallomics 1:458-470.
26. Mangiapia G, D'Errico G, Simeone L, Irace C, Radulescu A, et al. (2012) Ruthenium-based complex nanocarriers for cancer therapy. Biomaterials 33:3770-3782.

27. Simeone L, Mangiapia G, Irace C, Di Pascale A, Colonna A, et al. (2011) Nucleolipid nanovectors as molecular carriers for potential applications in drug delivery. Mol Biosyst 7:3075-3086.

28. Simeone L, Mangiapia G, Vitiello G, Irace C, Colonna A, et al. (2012) Cholesterol-based nucleolipid-ruthenium complex stabilized by lipid aggregates for antineoplastic therapy. Bioconjug Chem 23:758-770.

29. Mangiapia G, Vitiello G, Irace C, Santamaria R, Colonna A, et al. (2013) Anticancer cationic ruthenium nanovectors: from rational molecular design to cellular uptake and bioactivity. Biomacromolecules 14:2549-2560.

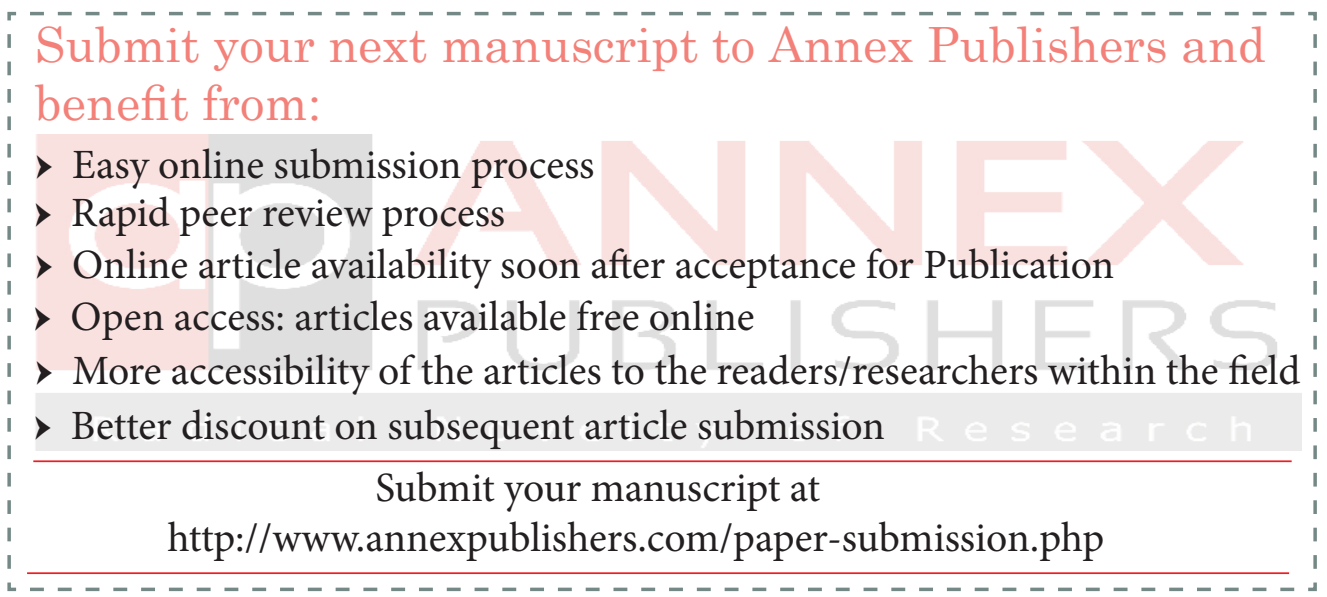

